\title{
Electrical Properties of Intermediate Band (IB) Silicon Solar Cells Obtained by Titanium Ion Implantation
}

\author{
Helena Castán*1), Eduardo Pérez ${ }^{1)}$, Héctor García ${ }^{1)}$, Salvador Dueñas ${ }^{1)}$, Luis \\ Bailón $^{1)}$, Javier Olea ${ }^{2)}$, David Pastor ${ }^{2)}$, Eric García-Hemme ${ }^{2)}$, Maite Irigoyen ${ }^{2)}$ and \\ Germán González-Díaz ${ }^{2)}$ \\ 1) Dept. de Electricidad y Electrónica, Universidad de Valladolid, ETSI Telecomunicación, Paseo de Belén 15, \\ 47011 Valladolid, Spain. *)electronic-mail: helena@ele.uva.es \\ 2) Dept. de Física Aplicada III (Electricidad y Electrónica), Facultad de Ciencias Físicas, Universidad \\ Complutense de Madrid, Av. Complutense s/n, 28040 Madrid, Spain
}

\begin{abstract}
Intermediate band silicon solar cells have been fabricated by Titanium ion implantation and laser annealing. A two-layer heterogeneous system, formed by the implanted layer and by the unimplanted substrate is obtained. In this work we present electrical characterization results which evidence the formation of the intermediate band on silicon when ion implantation dose is beyond the Mott limit. Clear differences have been observed between samples implanted with doses under or over the Mott limit. Samples implanted under the Mott limit have capacitance values much lower than the non-implanted ones as corresponds to a highly doped semiconductor Schottky junction. However, when the Mott limit is surpassed the samples have much higher capacitance, revealing that the intermediate band is formed. The capacitance increase is due to the big amount of charge trapped at the intermediate band, even at low temperatures. Titanium deep levels have been measured by Admittance Spectroscopy. These deep levels are located at energies which vary from 0.20 to $0.28 \mathrm{eV}$ bellow the conduction band for implantation doses in the range $10^{13}-10^{14}$ at $/ \mathrm{cm}^{2}$. For doses over the Mott limit the implanted atoms become non recombinant. Admittance measurements are the first experimental demonstration the Intermediate Band is formation. Capacitance voltage transient technique measurements prove that the

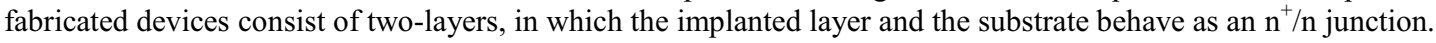

Keywords: Solar cells, intermediate band, ion implantation, admittance spectroscopy, capacitance-voltage transient technique.

PACS: 88.40.H-, 71.20.-b, 71.55.-i, 85.30. De

\section{INTRODUCTION}

The formation of an intermediate band IB in the midgap of a semiconductor has shown potential for drastically improving the efficiency of single junction solar cells [1]. This approach permits electrons of energy below the band gap to be pumped from the valence band into conduction band via two-photon absorption of lower energy that use this IB as an intermediary step.

IB solar cells can be created in a semiconductor from deep level impurities if their concentration is high enough.

Titanium Ti is a well-known deep impurity in $\mathrm{Si}$ which introduces a donor level. For instance, a level at $0.22 \mathrm{eV}$ below the conduction band has been detected by Hall effect experiments [2], although deep level transient spectroscopy studies pointed to a deeper position of the level about $0.29 \mathrm{eV}$ [3]. It is suggested that $\mathrm{Ti}$ atoms diffuse in silicon through a simple interstitial process according to electron paramagnetic resonance measurements of such samples [4]. These impurities are a well-known source of non radiative
Shockley-Reed-Hall SRH recombination. However, it has been argued that this recombination may be suppressed when the Ti concentration is high enough as to exceed the Mott transition $5 \times 10^{19} \mathrm{~cm}^{-3}$, and a band instead of a level of isolated impurities is formed [5].

It has been proved that the combination of different experimental techniques and the use of laser thermal processing allow the introduction of $\mathrm{Ti}$ atoms into the Si lattice in concentrations near or above the Mott limit, proving the possibility to form the IB in Si with this element [6].

In this work we use samples obtained in this way to prove the formation of intermediate band on silicon. Results obtained by Capacitance measurements, Admittance Spectroscopy and Capacitance-Voltage Transient Technique (CVTT) show the existence of active titanium deep levels and evidence the formation of the intermediate band. 


\section{SAMPLE FABRICATION}

$300 \mu \mathrm{m}$ silicon (111) n-type samples $(\mu=1450$ $\mathrm{cm}^{2} / \mathrm{Vs} ; \mathrm{n}=2.2 \times 10^{13} \mathrm{~cm}^{-3}$ at room temperature) were implanted in a IBS (Ion Beam Services) refurbished VARIAN CF3000 Ion Implanter at $32 \mathrm{KeV}$ with $\mathrm{Ti}$ at high doses $\left(10^{13}, 10^{14}\right.$ and $\left.10^{16} \mathrm{~cm}^{-2}\right)$. Then, the implanted Si samples were annealed by means of the Pulsed Laser Method (PLM) to recover the sample crystallinity. The PLM annealing process was performed by J. P. Sercel Associates Inc. (New Hampshire, USA). Samples were annealed with one 20 ns long pulse of a $\mathrm{KrF}$ excimer laser $(248 \mathrm{~nm})$ at energy densities from 0.2 to $0.8 \mathrm{~J} / \mathrm{cm} 2$. PLM is a highly non-equilibrium processing technique which is able to melt and recrystallize the Si surface up to about $100 \mathrm{~nm}$ deep in very short times $\left(10^{-8}-10^{-6} \mathrm{~s}\right)$. This rapid recrystallization time allows the incorporation of $\mathrm{Ti}$ atoms to the $\mathrm{Si}$ at concentrations well above the solubility limit for this element. Also, the PLM processing of the $\mathrm{Ti}$ implanted $\mathrm{Si}$ layer prevents secondary phase formation (i.e., Titanium silicide) even when the equilibrium solubility limit has been greatly exceeded.

Table 1 summarizes the samples fabrication process. Samples implanted with $10^{13}$ and $10^{14}$ at $/ \mathrm{cm}^{2}$ have Ti profiles bellow the Mott limit at any depth. In contrast, the sample implanted at a dose of $10^{16} \mathrm{at} / \mathrm{cm}^{2}$ shows impurity concentrations beyond the Mott limit up to a depth of $80 \mathrm{~nm}$. $\mathrm{TiCl}_{4}$ was used as Titanium precursor and the ion implantation energy was $32 \mathrm{keV}$. The implanted samples were annealed by means of the pulsed laser melting (PLM) method to recover the crystal lattice. Samples were annealed with one $20 \mathrm{~ns}$ long pulse of a $\mathrm{KrF}$ excimer laser $(248 \mathrm{~nm})$ at energy density of $0.6-0.8 \mathrm{~J} / \mathrm{cm}^{2}$.

TABLE 1. Samples description

\begin{tabular}{ccc}
\hline Sample & $\begin{array}{c}\text { Ti implanted dose } \\
\left(\mathbf{a t} / \mathbf{c m}^{\mathbf{2}}\right)\end{array}$ & $\begin{array}{c}\text { PL } \\
\text { Annealing }\end{array}$ \\
\hline Witness & None & No \\
UM1 & $10^{13}$ (Under Mott Limit) & Yes \\
UM2 & $10^{14}$ (Under Mott Limit) & Yes \\
OM & $10^{16}$ (Over Mott Limit) & Yes \\
\hline
\end{tabular}

\section{EXPERIMENTAL RESULTS}

\section{Capacitance - Voltage}

Clear differences between samples implanted with doses under or beyond the Mott limit. Figure 1 shows Capacitance-Voltage obtained at low temperature and $10 \mathrm{KHz}$. Samples implanted under the Mott limit have capacitance values much lower than the unimplanted one as corresponds to a metal-highly doped semiconductor Schottky junction. However, the samples with Intermediate Band have much higher capacitance due to the big amount of charge trapped at the intermediate band, even at low temperatures.

As ion implantation doses increase, silicon is more doped yielding a lowering of the Schottky barrier. In consequence, the capacitance value decreases. For doses beyond the Mott limit the Fermi level is pinned at the intermediate band, and a new barrier appears between the implanted and non-implanted regions and the total capacitance increases up to values much higher that for the non-implanted sample.

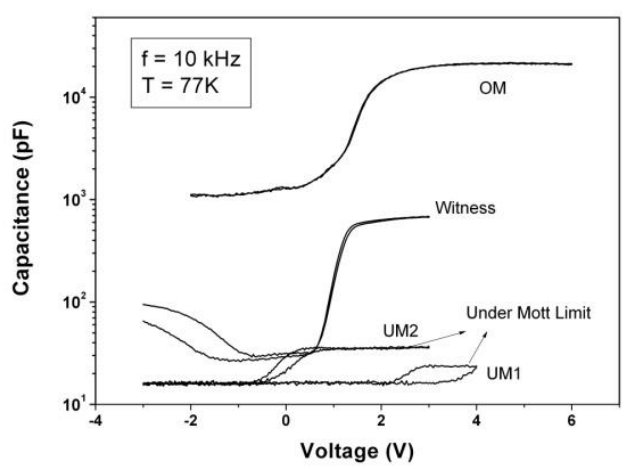

FIGURE 1. Capacitance-Voltage curves at $10 \mathrm{KHz}$ and low temperature.

\section{Admittance Spectroscopy}

Thermal admittance spectroscopy [7] is a technique which yields thermal emission rates of deep levels from the variation of capacitance and conductance of a junction as a function of temperature and frequency. These variations are due to the change in frequency of the measuring signal with respect to the time constant of charge and discharge processes of the deep levels. Each deep level existing in the semiconductor bandgap contributes with a peak in the conductance and an inflection point in the capacitance. Figure 2 summarizes the results obtained for all the samples studied in this work. We see that samples implanted under the Mott limit (UM1 and UM2) show conductance peaks as corresponds to the deep levels created by the implanted titanium species, whereas non implanted and over Mott (OM) limit implanted samples does not exhibit conductance peaks, in good agreement with the Intermediate Band (IB) theory [5], that predicts that deep levels become non recombinant when IB band has been formed. This is the first experimental evidence confirming that recombination is suppressed when the $\mathrm{Ti}$ concentration is high enough as to exceed the Mott limit. 


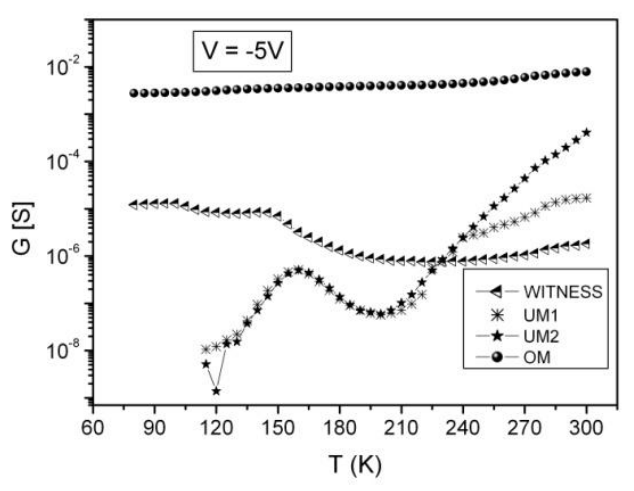

FIGURE 2. Conductance curves as a function of temperature at a frequency of $30 \mathrm{KHz}$.

In Figure 2 we can see that the conductance peak amplitude is the same for samples implanted at $10^{13}$ and $10^{14} \mathrm{at} / \mathrm{cm}^{2}$. That suggests that the concentration of recombinant deep levels reaches a saturation value. The remaining implanted atoms have a no recombinant nature. This nature is dominant when Mott transition occurs.

The conductance peak temperature is related to the deep level emission rate by the well-known equation:

$$
\boldsymbol{\theta}_{n}\left(T_{\text {peak }}\right)=\omega / 1.98
$$

where $\omega$ is the angular frequency. The emission rate follows an Arrhenius law, from which the energy location of the titanium deep level can be obtained. Figures 3 and 4 are the results obtained in this way for samples UM1 and UM2.

Although, the deep level energy is in the range of those elsewhere reported $[2,3]$, it varies depending on

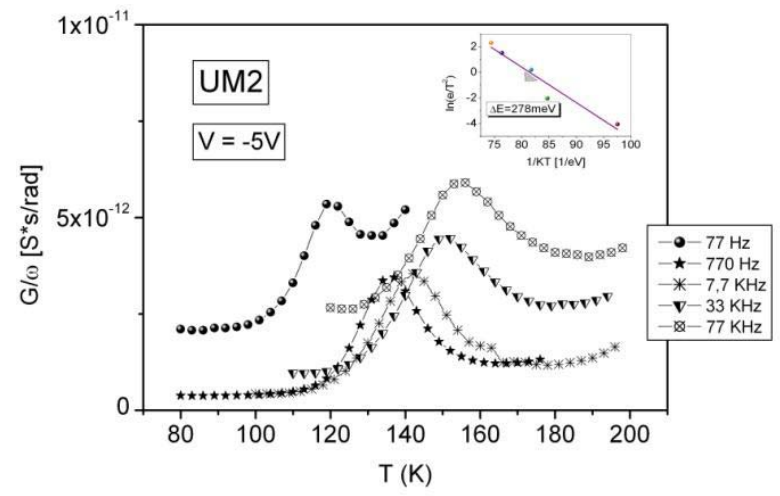

FIGURE 3. Conductance vs temperature at reverse bias of $5 \mathrm{~V}$ for a sample implanted at $10^{13} \mathrm{at} / \mathrm{cm}^{2}$

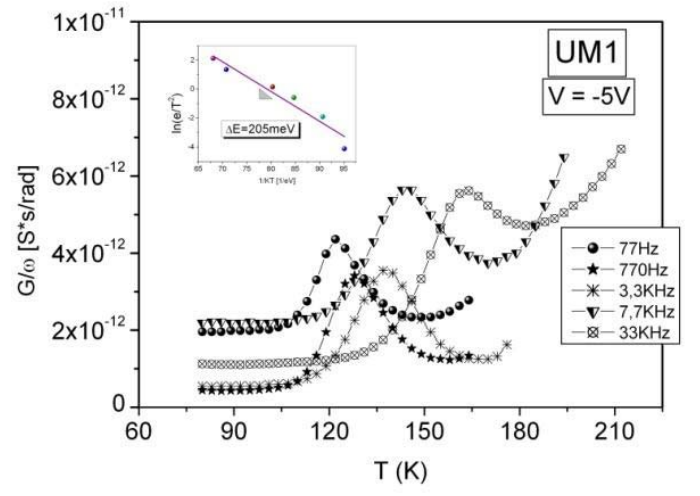

FIGURE 4. Conductance vs temperature at reverse bias of $5 \mathrm{~V}$ for a sample implanted at $10^{14}$ at $/ \mathrm{cm}^{2}$ :

the implantation doses. Deep levels are located at 205 $\mathrm{meV}$ under the conduction band for samples implanted at $10^{13} \mathrm{at} / \mathrm{cm}^{2}$ whereas it reaches a deeper value $(278$ $\mathrm{meV}$ ) for the $10^{14} \mathrm{at} / \mathrm{cm}^{2}$. In a previous work, Olea et al. [8] have proposed for OM sample an analytical two-layer model, in which the implanted layer and the substrate behave as an IB/n-Si type junction. They also deduce that the IB is located at $0.38 \mathrm{eV}$ below the conduction band and carriers at the IB behave as holes with a mobility of $0.4-0.6 \mathrm{~cm}^{2} \mathrm{~V}^{-1} \mathrm{~s}^{-1}$. This deeper energy value agrees with the trend of deep levels to reach deeper energies as doses increase.

\section{Capacitance-Voltage Transient Technique (CVTT)}

Among the different techniques used to determine doping profiles in semiconductors, the $\mathrm{C}-\mathrm{V}$ technique is one of the most extensively used. The $\mathrm{C}-\mathrm{V}$ technique has a greater sensitivity than that of the other techniques. In a previous work [9] we presented a technique based on $\mathrm{C}-\mathrm{V}$ that allows one to obtain both the profiles and emission activation energies for all deep centers present in the junction. This technique, named the capacitance-voltage transient technique (CVTT), basically consists of recording the instantaneous $\mathrm{C}-\mathrm{V}$ curve just after an emission pulse is applied to the junction.

We have obtained CVTT transients for samples UM1 and UM2, but these transients do not appear for "over Mott" samples since implanted species become non recombinant. Figure 5 illustrates CVTT transients for the sample implanted with a dose of $10^{14} \mathrm{at} / \mathrm{cm}^{2}$. We can see that at $200 \mathrm{~K}$ the capacitance transients increase with the emission time as is usual for a typical deep level. However, for high temperatures, the transients are anomalous. At $240 \mathrm{~K}$, a peak appears which moves towards more negative voltage as the 

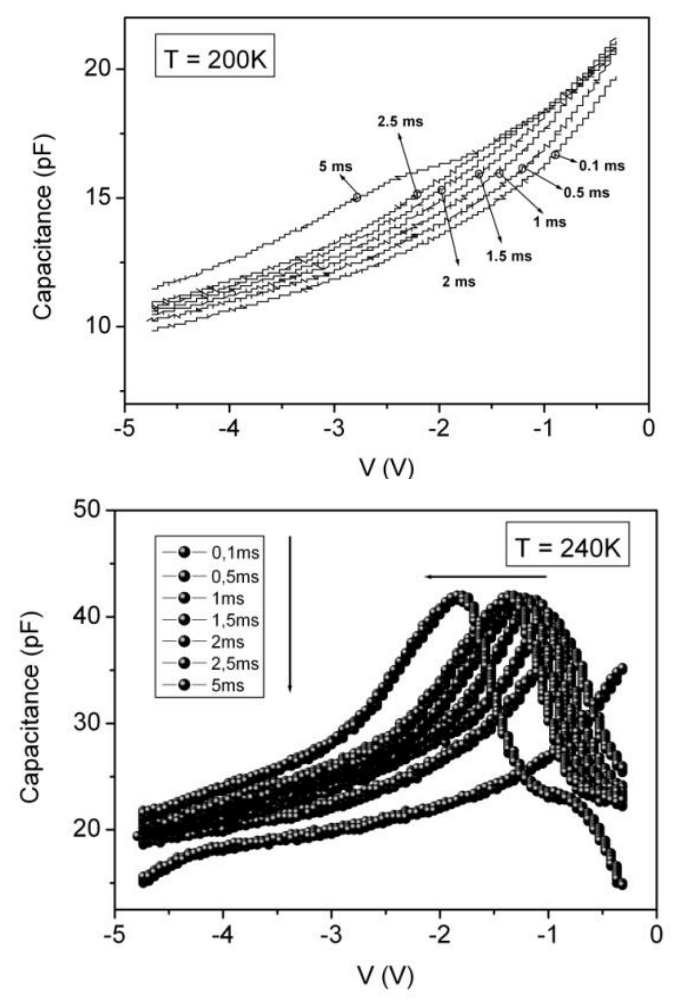

FIGURE 5. $1 \mathrm{MHz}$ CVTT results for a sample implanted at $10^{14} \mathrm{at} / \mathrm{cm}^{2}$ at two temperatures.

emission time increases. The implanted layer is just a highly doped semiconductor with a high deep level concentration. The entire device can be modeled as a Schottky diode in series with an $\mathrm{n}^{+} / \mathrm{n}$ homojunction. The Schottky diode is the one formed by the top metal and the implanted semiconductor layer. The $\mathrm{n}^{+}-\mathrm{n}$ junction is formed by the highly doped $n$ type implanted layer and the non-implanted substrate.

When a negative voltage is applied between top and bottom electrode, the Schottky diode is reversely biased and its capacitance, $C_{S}$, decreases with voltage. In contrast, a forward voltage is applied to the $\mathrm{n}^{+}-\mathrm{n}$ junction and its capacitance, $C_{J}$, increases with voltage. The measured capacitance results from the composition of the two in series capacitances:

$$
C_{M}^{-1}=C_{s}^{-1}+C_{J}^{-1}
$$

and its value is determined by the lower of the two capacitances. Then, $C_{S}$ is dominant for the most negative voltages, whereas at low voltages $C_{M}$ is determined by $C_{J}$. A maximum on the measured capacitance appears that indicates the transition from the $C_{S}$-dominated to the $C_{J}$-dominated regime. In Figures 5(b) we can see that the transition peak varies with the emission pulse duration, $t_{e m}$. It also varies with temperature (not shown). Moreover, the total voltage is differently shared between the two junctions: when $C_{S}$ increases the voltage drop on the $\mathrm{n}^{+}-\mathrm{n}$ junction also increases. That is the reason why curves seem to move with emission time while preserving the same shape at the right side of the transition peak.

\section{CONCLUSIONS}

Intermediate band silicon solar cells have been fabricated by Titanium ion implantation and laser annealing. $\mathrm{C}-\mathrm{V}$ measurements show clear differences between samples implanted with doses under or beyond the Mott limit. Admittance Spectroscopy evidence that when the titanium ion implantation overcomes the Mott limit, the deep centers do not show conductance peaks. This is the first experimental evidence confirming that recombination is suppressed when the Ti concentration is high enough as to exceed the Mott limit. CVTT measurememts on samples under the Mott limit shows a peculiar behavior which allows these devices to be modeled as a Schottky junction in series with an $\mathrm{n}^{+} / \mathrm{n}$ homojunction.

\section{ACKNOWLEDGMENTS}

This work was partially supported by VA128A11-2 funded by the Junta de Castilla y León, Spanish TEC2011 under grant 27292-C02-01, S/2009/ENE1477) funded by the Comunidad de Madrid and CSD2006-00004 funded by the Spanish Consolider National Program. Research of E. Perez was supported by a University of Valladolid FPI grant and research of E. Garcia-Hemme by a PICATA predoctoral fellowship of the Moncloa Campus of International Excellence (UCM-UPM).

\section{REFERENCES}

1. A. Luque and A. Martí, Phys. Rev. Lett. 78, 5014(1997).

2. J.-W. Chen, A. G. Milnes, and A. Rohatgi, Solid-State Electron. 22, 801 (1979).

3. D.Mathiot and S.Hocine, J. Appl. Phys. 66, 5862 (1989).

4. S. Hocine and D. Mathiot, Appl. Phys. Lett. 53, 1269 (1988).

5. A. Luque, A. Martí, E. Antolín, and C. Tablero, Physica B 382, 320 (2006).

6. J. Olea, M. Toledano-Luque, D. Pastor, G. GonzálezDíaz and I. Mártil, J. Appl. Phys. 104, 016105, (2008)

7. J. Barbolla, S. Dueñas and L. Bailón, Solid-State Electron. 35(3), 285-297 (1992)

8. J. Olea, G. González-Díaz, D. Pastor, I. Mártil, A. Martí, E. Antolín and A. Luque. J.Appl. Phys. 109, 063718 (2011)

9. S. Dueñas, H. Castán, L. Enríquez, J. Barbolla, J. Montserrat and E. Lora-Tamayo. Semicond. Sci. Technol. 9, 1637-1648 (1994). 
Copyright of AIP Conference Proceedings is the property of American Institute of Physics and its content may not be copied or emailed to multiple sites or posted to a listserv without the copyright holder's express written permission. However, users may print, download, or email articles for individual use. 